\title{
Dispositivos digitales en el hogar: incidencia de las desigualdades y las políticas públicas de acceso partir de un análisis multivariado.
}

\section{Digital devices in households: the influence of inequalities and public access policies based on a multivariate analysis.}

\author{
Ana Rivoir*, Santiago Escuder**
}

*Universidad de la República, Uruguay.

** Universidad de la República, Uruguay.

Resumen

\begin{abstract}
Las políticas de inclusión digital en América Latina buscan reducir la brecha digital para contribuir a la reducción de las desigualdades sociales. En el artículo se analiza la infraestructura digital de los hogares uruguayos y su relación con su condición socioeconómica y variables demográficas. Se realiza un análisis del alcance de las desigualdades de acceso a las tecnologías tanto analógicas como digitales y la incidencia de las políticas públicas con la reducción de la brecha digital. Se describen las características de los hogares beneficiados por las políticas de inclusión digital y qué sectores de la población permanecen fuera de su alcance. A partir de la Encuesta de Usos de Tecnologías de la Información y la Comunicación (EUTIC 2013) del Instituto Nacional de Estadísticas de Uruguay (INE), se realiza un análisis multivariado para establecer cuáles son los conjuntos de variables que determinan factores relevantes así como definir posibles conglomerados y tipología de hogares. Se encuentra que persiste un vínculo estrecho con la desigualdad de ingreso y la brecha de acceso en los hogares y un efecto positivo de las políticas TIC aunque con un techo para alcanzar los hogares más excluidos.
\end{abstract}

Palabras clave: desarrollo humano; infraestructura digital, políticas públicas

Abstract

Digital inclusion policies in Latin America seek to reduce the digital divide as a way to contribute with the reduction of social inequalities. In the research, we analyze the information infrastructure of Uruguayan households and its relationship with their socioeconomic status. We analyze the inequalities in access, the process from analogical to digital technologies and the impact of public policies in the reduction of the digital divide. Going through a description and characterization of households benefited of digital inclusion policies and of the people that remain excluded from technologies. Based on the Information and Communication Technologies Usage Survey (EUTIC 2013) of the National Statistics Institute of Uruguay (INE), we carried out a multivariate analysis to establish which sets of variables determine relevant factors and define clusters and typology of households. Overall, we conclude that there is a close link between income inequalities, and that the access gap of some households persists, there is a positive effect of ICT policies but with important limitations to reach the most excluded households.

Keywords: human development; digital infrastructure; public policy

\section{Desarrollo humano informacional, infraestructura y políticas}

El modo de desarrollo informacional sustentado en el desarrollo y expansión de las Tecnologías de la Información y la Comunicación (TIC) desafía las estrategias de desarrollo en las sociedades contemporáneas, e interpela su conceptualización. Lo que Manuel Castells (2000) ha denominado Sociedad Red, refiere a la estructura organizacional cuyo nervio de conducción son los flujos de Internet. Esto habilita la difusión de información y conocimiento a tiempo real a nivel global y gesta la economía basada en conocimiento. Las posibilidades de crecimiento económico de las sociedades, se sustentan en la 
capacidad de adaptación a este nuevo modo de desarrollo capitalista. Es así que las economías de los países, para ser competitivas, deben crear valor agregado en conocimiento. Necesitan crear capacidad tecnológica y capacidades de uso para su aprovechamiento.

En continuidad con este desarrollo teórico, Castells y Himanen (2014) buscan establecer el vínculo del profundo cambio tecnológico con el concepto de desarrollo humano, abandonando el economicismo para comprender la complejidad del concepto. Entienden que el desarrollo humano se establece en la relación entre la producción material, el bienestar humano y la organización sociocultural. Afirman que si prima el desarrollo informacional, se tiende a consolidar las redes del capitalismo financiero global mientras que iniciativas que sólo apunten al desarrollo humano sin transformación informacional, conducen a sistemas no sustentables y crisis fiscal. Concluyen así que el desafío de las estrategias contemporáneas, es vincular al desarrollo informacional con el desarrollo humano en su expansión conjunta.

Del análisis de los antecedentes de investigación en sociedades latinoamericanas, se desprende que los países con desarrollo digital son los que logran desarrollo económico. Estos resultados confirman el vínculo existente entre el desarrollo digital y el crecimiento económico. (Peres y Hilbert, 2009; Katz, 2009) También se evidencia que esta digitalización relaciona positivamente no sólo con el crecimiento económico sino también con la disminución de la tasa de desempleo. (Katz, 2013) En este contexto, las brechas de acceso se conforman como un factor que contribuye cada vez más a aumentar desigualdades entre países, regiones y personas.

En este sentido, la necesidad de políticas en esta área, ha sido profusamente fundamentada por diversos estudios y autores (Gascó et al, 2007; Peña - López, 2009; Guerra y Jordán, 2010). Se sostiene que son fundamentales las acciones y políticas específicas para que la expansión de las TIC contribuya con el desarrollo humano así como con la reducción de las desigualdades. Varias son las agencias internacionales (UIT, 2014; CEPAL, 2012) que recomiendan la formulación e implementación de estrategias digitales, de políticas TIC en distintos ámbitos como la educación formal como no formal o políticas de acceso mediante distintas iniciativas sociales o comunitarias.

En este contexto resulta central cuestionarse acerca de cuáles son las iniciativas más efectivas para la reducción de las desigualdades digitales en contexto de países donde el acceso vía el mercado resulta muy dificultoso para grandes sectores de la población como lo son los países latinoamericanos.

La brecha de acceso en América Latina sigue siendo un problema, sobre todo en los países de menores ingresos y menos desarrollo relativo, como lo evidencian las distintas estadísticas a nivel mundial ${ }^{1}$.

Esta desigualdad en el acceso a TIC se encuentra en estrecha relación con las desigualdades sociales existentes en cada sociedad en concreto e interactúa en forma negativa para el desarrollo humano pues limita las oportunidades para vastos sectores de la población. (Mansell, 2002; Hargittai, 2004) Estudios realizados confirman que la reducción de estas desigualdades, así como aumentar las capacidades para el desarrollo humano y digital, tiene que ver con muchos factores. Entre los elementos más importantes, se encuentran las características geográficas de cada país o región, su mercado de telecomunicaciones y la capacidad institucional con la que cuenta cada sociedad para el desarrollo de políticas públicas en la materia. (Katz, 2009)

Dentro de los elementos encontrados para la expansión del uso de la banda ancha en América Latina, Katz y Galperin (2013) registran dos caminos. Por un lado, el acceso vía el mercado apostando a la reducción 
de precios que a mediano y largo plazo facilite la adquisición de los mismos por parte de sectores más amplios de la población. Por otro, las iniciativas de política pública orientadas a reducir estas desigualdades que pueden asumir distintas características. Algunas son exclusivamente estatales, otras conjugan el sector público y el privado o los subsidios a la oferta. Dentro de este último tipo de estrategias es que ubican las políticas públicas implementadas en Uruguay en la última década.

Dado el contexto de comprender que la expansión del desarrollo digital es un componente central para el desarrollo económico de las sociedades, es necesario que el acceso alcance a la mayor cantidad de sectores de la sociedad. En este sentido, emerge la pregunta acerca de cuáles son los elementos y componentes de estas políticas y cuanto han logrado expandir el acceso y conectividad entre la población en sociedades desiguales como lo son las latinoamericanas.

La Comisión Económica Para América Latina (CEPAL, 2013) indica que el ecosistema de la economía digital, tiene como componente último el usuario final, compuesto por individuos, empresas y gobierno. Señala que las desigualdades en la distribución del ingreso y el acceso a servicios públicos son centrales y condicionan el acceso y uso de internet. Asimismo, las dificultades del acceso en el hogar y en el lugar de trabajo son factores claves para alcanzar al usuario final y por tanto que influyen en forma importante en la conformación del ecosistema. Sostiene que para esta transición hacia sociedades informacionales se requiere de la infraestructura y el equipamiento necesario como factor primordial. Si bien no constituye el único elemento, es imprescindible para su desarrollo. Implica inversión y transformación en los distintos sectores y ámbitos como las empresas, el territorio y los hogares.

Interesa por tanto conocer cuáles son las condiciones de infraestructura que habilitan cambios y facilitan la transición hacia sociedades basadas en el desarrollo informacional y en el conocimiento. Se requiere conocer las condiciones de infraestructura digital de los hogares y la existencia y tipos de hogares en etapa analógica y digital. Dado que estas transformaciones implican un proceso de cambio, interesa saber si se pueden categorizar los como hogares según su equipamiento para conocer el estadio en el que se encuentran. Puede ser que estén en una etapa analógica, en transición o ya hayan ingresado a pleno en la etapa digital.

A tales efectos, en este trabajo se describe la infraestructura informacional de los hogares uruguayos, según su equipamiento y condiciones de acceso a Internet. Este dato es importante pues existe evidencia de que el acceso en el hogar favorece el uso, la frecuencia y la intensidad del mismo (CEPAL, 2013). Se trata de un factor que está cambiando debido a la aparición de los dispositivos móviles pero que continúa siendo un dato de referencia.

Se busca con el presente análisis, conocer la relación entre la infraestructura de los hogares y las variables económicas. Es decir, cual es la relación entre las variables estructurales (sociales y económicas) y el acceso a tecnología para poder determinar el vínculo entre la condición social de los hogares y su acceso a los dispositivos y recursos de las tecnologías digitales dando cuenta del alcance de las desigualdades y sus características de incorporación al desarrollo informacional y al desarrollo humano.

A su vez, detectar la incidencia y contribución de las políticas públicas en este proceso y su relación con la reducción de la brecha digital de acceso, es posible a partir del conociendo de las características de quienes han beneficiado de las políticas de inclusión digital en particular y qué sectores de la población permanecen fuera de su alcance. 


\section{Metodología}

Para conocer las características de la infraestructura digital de los hogares se propuso explorar y analizar los diferentes factores asociados a la tenencia de dispositivos TIC y para ello se realizó un análisis multivariado. Esto permite establecer cuáles son los conjuntos de variables que determinan factores, así como definir posibles conglomerados de hogares.

La técnica para la exploración de variables categóricas cualitativas fue el Análisis de Correspondencias Múltiples (ACM) que permite reducir la complejidad estadística univariada, al igual que construir y jerarquizar factores explicativos (López Roldan y Fachelli 2013). El objetivo principal de esta técnica fue aglomerar las condiciones estructurales y de acceso a dispositivos TIC. Se utilizó el método de Ward para la elaboración de los clusters de hogares y así determinar perfiles de acceso. ${ }^{1}$

La fuente de datos es la Encuesta de Usos de Tecnologías de la Información y la Comunicación (EUTIC 2013) del Instituto Nacional de Estadísticas de Uruguay (INE), conformada por unos 3646 hogares que ponderados y expandidos en el país representan 1.003.451. Es representativa a nivel nacional para el país urbano, pues incluye la capital de Uruguay (Montevideo), área metropolitana y las capitales y localidades de más de 5000 habitantes del resto de los diecinueve departamentos del país.

Se tomaron los quintiles de ingreso per cápita para determinar el estrato socio-económico de pertenencia de los hogares así como el estrato muestral de dichos hogares (bajo, medio bajo, medio, medio alto, alto). Para seleccionar la región del hogar se tomó la codificación original de la encuesta, la cual define dos regiones: "Montevideo" e "Interior urbano".

Se seleccionaron variables que comprenden los distintos dispositivos TIC que se encuentran en el hogar que se presentan en el Cuadro $\mathrm{N}^{\circ} 1$.

1 El método de Ward tiene como ventaja la formación de clases compactas, de igual tamaño, consistentes internamente, con la mayor diferencia entre grupos y con mínima pérdida de información La solución arrojo también siete y ocho clusters de hogares. 
Figura 1: Variables utilizadas en el análisis según la EUTIC 2013

\begin{tabular}{ccc}
\hline \multicolumn{1}{c}{ Teléfonos/Radios } & TV & PC y conexión a Internet \\
\hline Teléfono de línea & TV de tubo & PC de escritorio \\
Radio convencional & TV plasma, LED o LCD & Laptop o similar \\
Radio integrada a otro dispositivo & TV integrada a otro dispositivo & Tableta \\
Radio a través de Internet & TV a través de otros medios & Computadora de Ceibal \\
Radio a través de otros medios & TV Abierta & Línea discada \\
& TV para Abonados & Banda Ancha \\
& TV Satelital & Fibra óptica \\
& TV por Internet & Conexión Plan Ceibal \\
& TV a través de otras señales & Internet Móvil \\
& & Acuerdo con un vecino \\
\end{tabular}

Fuente: Selección propia de las variables de la EUTIC 2013.

El interés del análisis está centrado en la infraestructura de los hogares y por lo tanto no se incorpora el uso en centros comunitarios o de los teléfonos celulares en forma específica. Al momento de la encuesta un $25,8 \%$ disponía de un teléfono móvil inteligente (smartphone) y el análisis de esta categoría se realiza a través de lo que en la EUTIC de denomina "Internet móvil". La encuesta no incluye preguntas específicas sobre algunos programas y políticas como "Hogares universales" de la empresa estatal de telecomunicaciones (Antel) por lo que su influencia debió ser puesta a prueba en el análisis.

\section{La infraestructura digital de los hogares uruguayos: capacidades para el desarrollo informacional}

Uruguay evidencia un desarrollo digital que se destaca en la región latinoamericana. Según datos de la Unión Internacional de Telecomunicaciones (UIT, 2014), 61,5 de cada 100 habitantes en el país, son considerados usuarios de Internet, sólo por detrás de Chile (72,4\%), y Argentina (64,7\%). Es el cuarto país de las Américas con un Índice de Desarrollo Tecnológico (IDT) considerado alto (5,92). La asequibilidad a la Internet de banda ancha tan sólo representa el 1,63\% del total de Ingresos de los Hogares.

La estrategia de desarrollo informacional desplegada en el país desde el 2005, se integró con una estrategia de desarrollo humano. Asumió este enfoque de desarrollo informacional con desarrollo humano y con esa orientación se implementaron políticas públicas. Estas iniciativas se desplegaron en un contexto de crecimiento económico y de ejecución de diversas políticas sociales dirigidas a mejorar las condiciones de vida de la población. La reducción de la pobreza y la mejora de distintos indicadores sociales. La 
principal limitación de esta estrategia se evidencia en las dificultades del país para cambiar la estructura productiva hacia una economía basada en conocimiento. Esto pone en duda la sustentabilidad del proceso así como la incorporación de los sectores más excluidos al mismo y la necesidad urgente de expansión del paradigma digital a los distintos ámbitos. (Rivoir, 2013; Rivoir, 2016)

Las estrategias desarrolladas incluyen la elaboración e implementación de la Agenda Digital Uruguay (ADU) a cargo de la Agencia de Gobierno Electrónico para la Sociedad de la Información y el Conocimiento (AGESIC, http://www.agesic.gub.uy/) de la Presidencia de la República (http://www.presidencia.gub.uy). Se trata de una hoja de ruta que nuclea distintas iniciativas gubernamentales y sociales orientadas al desarrollo digital y humano. En cuanto a las políticas sectoriales, dentro de las de mayor impacto se encuentra el Plan de Conectividad Educativa de Informática Básica para el Aprendizaje en Línea (Plan Ceibal, http://www.ceibal.edu.uy). Este consiste en una adaptación local del proyecto One Laptop per Child del Instituto Tecnológico de Massachusetts. Se implementó a partir del sistema de educación pública a nivel de educación primaria y secundaria, así como formación docente. Implica la entrega de un dispositivo tecnológico a cada niño, niña y docentes de la educación pública a nivel de primaria y secundaria, así como iniciativas en torno a contenidos, apoyo a la enseñanza, formación de docentes, entre otras acciones. (Rivoir \& Lamschtein, 2014)

Otras políticas como los 129 telecentros del Ministerio de Educación y Cultura (http://www.centrosmec.gub.uy/) o el Programa Universal Hogares (https://www.infouruguay.com.uy/ADSL/ADSL-UNIVERSAL-HOGARES.htm) de tarifa reducida y la extensión de la fibra óptica por parte de la empresa pública de telecomunicaciones (ANTEL, www.antel.com.uy), han buscado contribuir a reducir la brecha de acceso y uso. Esta última política provee de 1 giga byte de conexión a Internet a todos los hogares que accedan a telefonía fija sin excepción.

Por último, se ha iniciado y se encuentra en ejecución desde 2015 una política pública nacional, orientada a brindar acceso a las personas mayores. Se trata del Plan Ibirapitá (http://ibirapita.org.uy/), su incidencia en la infraestructura y en la brecha digital podrá ser contrastada a medida que avance y estén disponibles los datos estadísticos. Consiste en la entrega de tablets con conexión a Internet y un sistema operativo adecuado a las personas mayores. Beneficia a aquellos con jubilaciones más bajas y se brinda un taller de capacitación para favorecer el uso.

\section{Características generales}

Un primer aspecto que vincula el desarrollo digital con las políticas implementadas hasta la fecha de la encuesta, es la constatación del aumento en el acceso por a TIC e Internet por parte de los hogares. De acuerdo a análisis anteriores han mejorado sostenidamente en la década 2006 - 2015 los distintos indicadores. El porcentaje de hogares con computadora aumenta del 19,1\% en 2006 a 67,4\% en 2014 y el acceso a Internet de 9,7\% a 57,4\% (ENHV, 2006 y 2014). En el 2013 y según resultados de la EUTIC para dicho año, el 70\% de los hogares disponían al menos de algún tipo de computadora en el hogar y un $65 \%$ algún tipo de conexión a Internet. Es de interés conocer cuál es la relación de estos cambios tecnológicos 
con otras características de los hogares y cuáles son sus potencialidades y cuales los factores con las que se relacionan positivamente.

Como se muestra en el Gráfico №1, la mayoría de los hogares disponen de bienes analógicos. Un 91,8\% accedía a radio convencional, $81,8 \%$ a TV de tubo, y un $71,1 \%$ disponía de telefonía fija. Como complemento de estos dispositivos, $63.4 \%$ accedía a la señal de TV abierta y un 63,2\% de los hogares a TV para abonados. No es menor la cantidad de hogares que acceden a TV plasma, LED o LCD, aunque representan poco más de la mitad de los que disponen de TV por tubo tradicional. Son muy pocos los hogares que integran servicios de Internet a estos dispositivos. Tan sólo $5 \%$ ve TV por Internet, pese a que $23,8 \%$ escuchan radio a través de la red. Se releva que un $83 \%$ de los integrantes de estos hogares utiliza celular y un $25,8 \%$ disponía de un teléfono móvil inteligente (smartphone) (EUTIC, 2013).

Gráfico 1: Tenencia de diferentes dispositivos en el hogar, año 2013. Medido en porcentaje de hogares para todo el país.

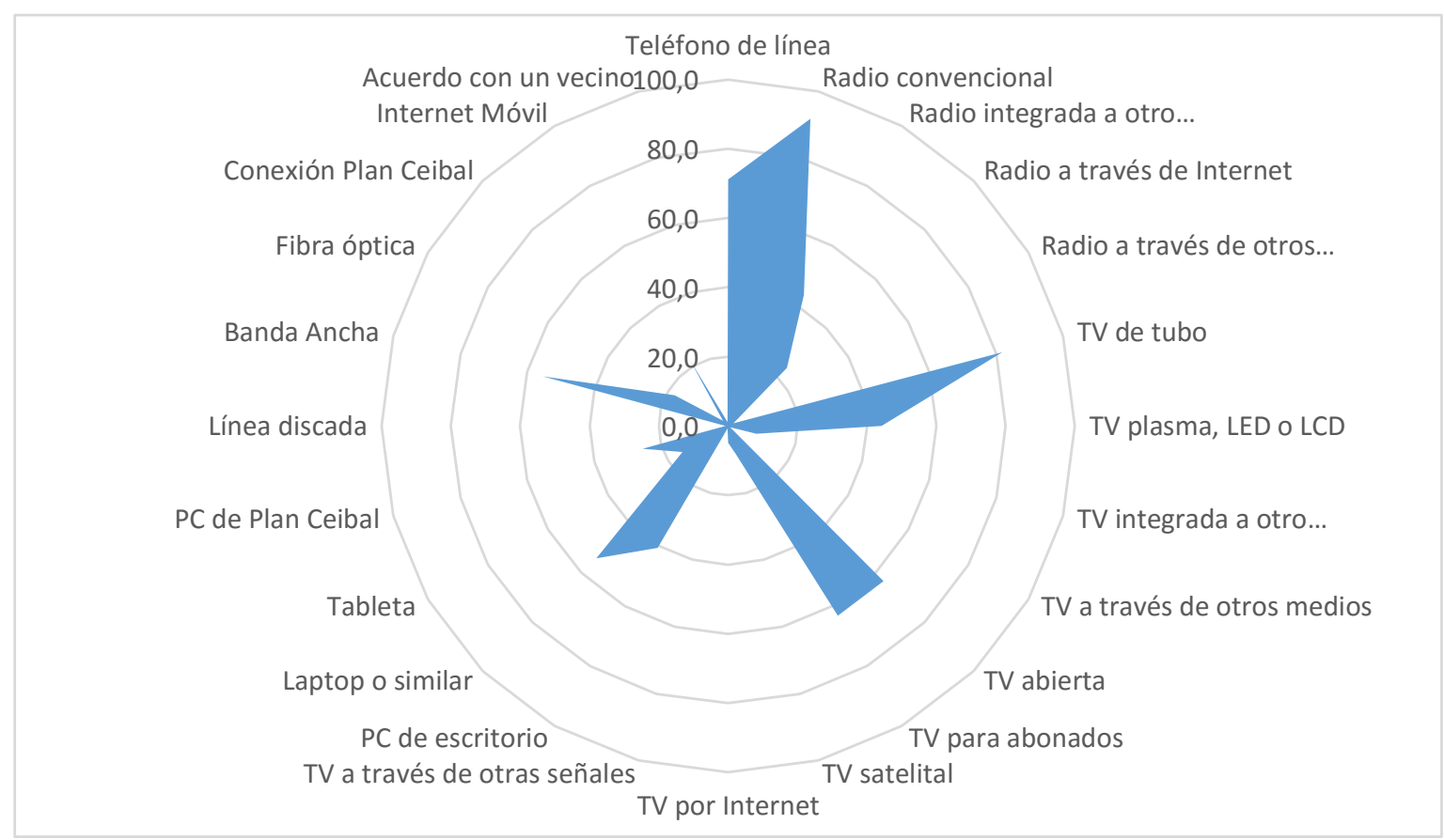

Fuente: Procesamiento propio de EUTIC 2013.

En primer lugar, se analizan las características tecnológicas de los hogares uruguayos. El 53,9\% de los hogares accedía a laptop, 40,6\% a computadora de escritorio. Sumado a la tenencia de "tableta" (15,2\%), el porcentaje de hogares que dispone de dispositivos portátiles que no son de Ceibal, alcanza el 69,1\%. El porcentaje de hogares que accede al laptop del Ceibal (política pública) alcanza al 25,6\%, lo que constituye un porcentaje significativo para el aumento tan pronunciado en el acceso. Sólo el 2,1\% accede a Internet por señal de Ceibal lo que constituye un alcance muy limitado y da cuenta que esta política no está influyendo en el acceso a internet de los hogares.

El 55,3\% de los hogares cuenta con conexión a Internet por banda ancha y un $21,8 \%$ a Internet móvil. Sin embargo, si se trata de banda de mayor velocidad como la fibra óptica, tan solo un $17,8 \%$ contrata este servicio. 
Tomando en cuenta el cruce de aquellos hogares que tienen algún tipo de conexión a Internet (ADSL, fibra óptica, línea discada, etc.), con aquellos sin conectividad, se evidencia que la infraestructura de los hogares está muy segmentada. Es así que en aquellos hogares que tienen conexión a Internet, la tenencia de teléfono fijo alcanza a un $86,3 \%$ y $56,7 \%$ la tenencia de algún TV plasma, LED o LCD. En aquellos hogares que no disponen de conectividad estos porcentajes descienden al $46,8 \%$ y $19,4 \%$ respectivamente.

Gráfico 2: Tenencia de diferentes dispositivos en el hogar según tenencia de conexión a Internet, año 2013. Medido en porcentaje de hogares para todo el país.

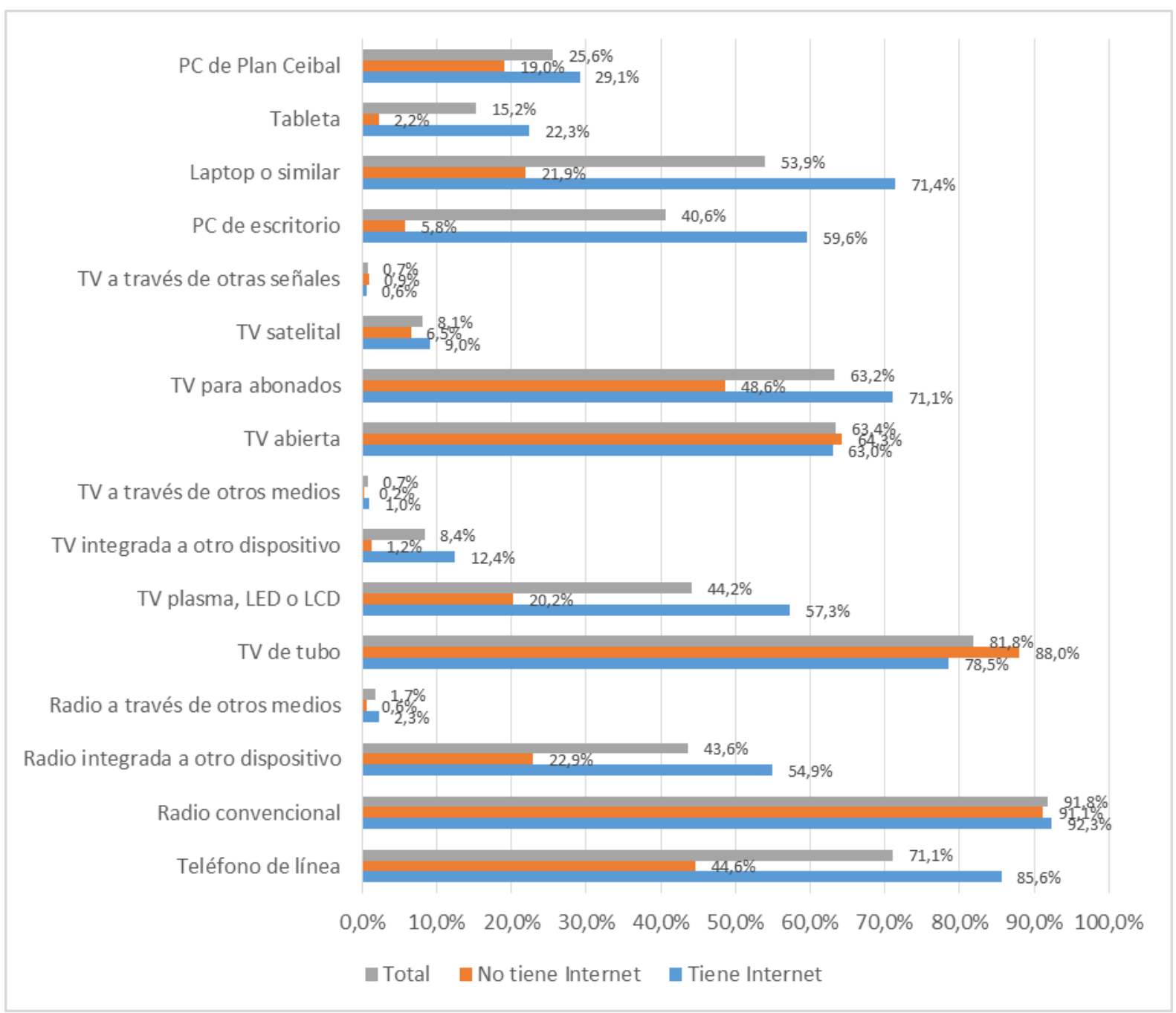

Fuente: Procesamiento propio de EUTIC 2013.

En este sentido, la tenencia o no de Internet puede definir distintos tipos de hogar. Por un lado, aquellos hogares que tienen los dispositivos e implementos producto del desarrollo de la tecnología digital como TV Plasma, computador y algún tipo de conectividad a Internet. Se trata de hogares "integrados" al paradigma informacional. Según datos de la EUTIC representan un 32\% de los hogares.

Por otro lado, se encuentran los hogares que cuentan con los mismos dispositivos digitales pero sin 
conexión a Internet. Estos hogares se podrán definir como "transicionales". Es decir, tienen la infraestructura y condiciones para la conectividad pero no acceden a Internet. Representan un $25,4 \%$ de los hogares.

Por último, están aquellos hogares sin acceso y sin dispositivos digitales, es decir se encuentran excluidos y representan casi un $11 \%$ del total.

\section{Análisis multidimensional. Principales factores.}

Para conocer los factores principales se realizó un análisis de las diez principales dimensiones. En los dos principales factores se encuentra el $85,1 \%$ de la inercia de los datos explicada, por lo que el análisis factorial se puede reducir principalmente a dos dimensiones.

El Cuadro N²2 detalla a continuación los factores para el Análisis de Correspondencia Múltiple (ACM).

Cuadro 2: Principales factores según análisis factorial.

\begin{tabular}{cccccc}
\hline Factor & Valor propio & \% Inercia & Valor propio corregido $\left(^{*}\right)$ & \% Inercia (1) & \% Inercia (2) \\
\hline 1 & 0,223905 & $13,43 \%$ & 0,034950 & $73,99 \%$ & $50,97 \%$ \\
2 & 0,115842 & $6,95 \%$ & 0,005437 & $11,51 \%$ & $7,93 \%$ \\
3 & 0,096712 & $5,80 \%$ & 0,002884 & $6,10 \%$ & $4,21 \%$ \\
4 & 0,080709 & $4,84 \%$ & 0,001364 & $2,89 \%$ & $1,99 \%$ \\
5 & 0,074598 & $4,48 \%$ & 0,000932 & $1,97 \%$ & $1,36 \%$ \\
6 & 0,064181 & $3,85 \%$ & 0,000385 & $0,81 \%$ & $0,56 \%$ \\
7 & 0,060223 & $3,61 \%$ & 0,000239 & $0,51 \%$ & $0,35 \%$ \\
8 & 0,057875 & $3,47 \%$ & 0,000169 & $0,36 \%$ & $0,25 \%$ \\
9 & 0,057707 & $3,46 \%$ & 0,000165 & $0,35 \%$ & $0,24 \%$ \\
10 & 0,057340 & $3,44 \%$ & 0,000155 & $0,33 \%$ & $0,23 \%$ \\
\hline
\end{tabular}

Fuente: Procesamiento propio de la EUTIC 2013.

El primer factor, explica prácticamente un $74 \%$ de la inercia de los datos. Se le denominó "capital económico" pues se nutre por la tenencia de dispositivos analógicos y digitales, excluyendo la tenencia de equipos proporcionados por Ceibal. Entre estos elementos a estas contribuciones se destaca el teléfono fijo con un 6,88\%, TV plasma, LED o LCD con 8,03\%, PC de escritorio 5,16\%, Tablet 5,40\%. La tenencia de diferentes tipos de banda ancha nutre también este factor. La tenencia de conexión de Internet por banda ancha explica el $13,42 \%$ de la contribución al primer factor, y la fibra óptica 7,49\%. 
La tenencia de un dispositivo proporcionado por Ceibal, contribuye a nutrir el segundo factor por lo que se le denominó factor de "Política TIC". Solamente contribuyen sustantivamente a este factor la posesión de elementos del Ceibal (27,88\%), como el acceso a un dispositivo o a su señal de Internet en el hogar $(5,91 \%)$. Este segundo factor no logra hacer una diferencia sustancial en la tenencia de infraestructura de los hogares ya que sólo explica sólo el $11 \%$.

En el gráfico $\mathrm{N}^{\circ} 3$ se presenta el ACM factorial y se muestra las variables y dicha correlación. Este permite ver cómo la tenencia de dispositivos, se encuentra muy asociada a los quintiles de ingreso per cápita (primer factor). También con otras variables como la región Montevideo- Interior urbano, evidencia la mejor situación de los hogares de la capital frente los del resto del país. Da cuenta de una brecha territorial a la vez que en la capital también se encuentra fragmentado el acceso TIC.

El eje de ordenadas " $Y$ " configura el efectos de las políticas TIC a través de la tenencia del laptop XO en el hogar, así como la conectividad de la red Ceibal. Estas diferencias no logran "estirar" el mapa de correspondencias, básicamente influido por el primer factor, no logra incidir significativamente en la tenencia de infraestructura TIC de los hogares.

Por otro lado, en la abscisa " $X$ " donde recaen los efectos del primer factor, las diferencias son mayores. En los cuadrantes comprendidos en el eje - $X$ se encuentran los hogares más pobres y menor provistos de infraestructura TIC. En los cuadrantes comprendidos en los ejes $+X$ se encuentran aquellos hogares de los quintiles con mayores ingresos, preferentemente de la capital del país, y con mayor diversificación en la infraestructura TIC. 
Gráfico 3: Tenencia de diferentes dispositivos en el hogar según el espacio factorial y de ACM.

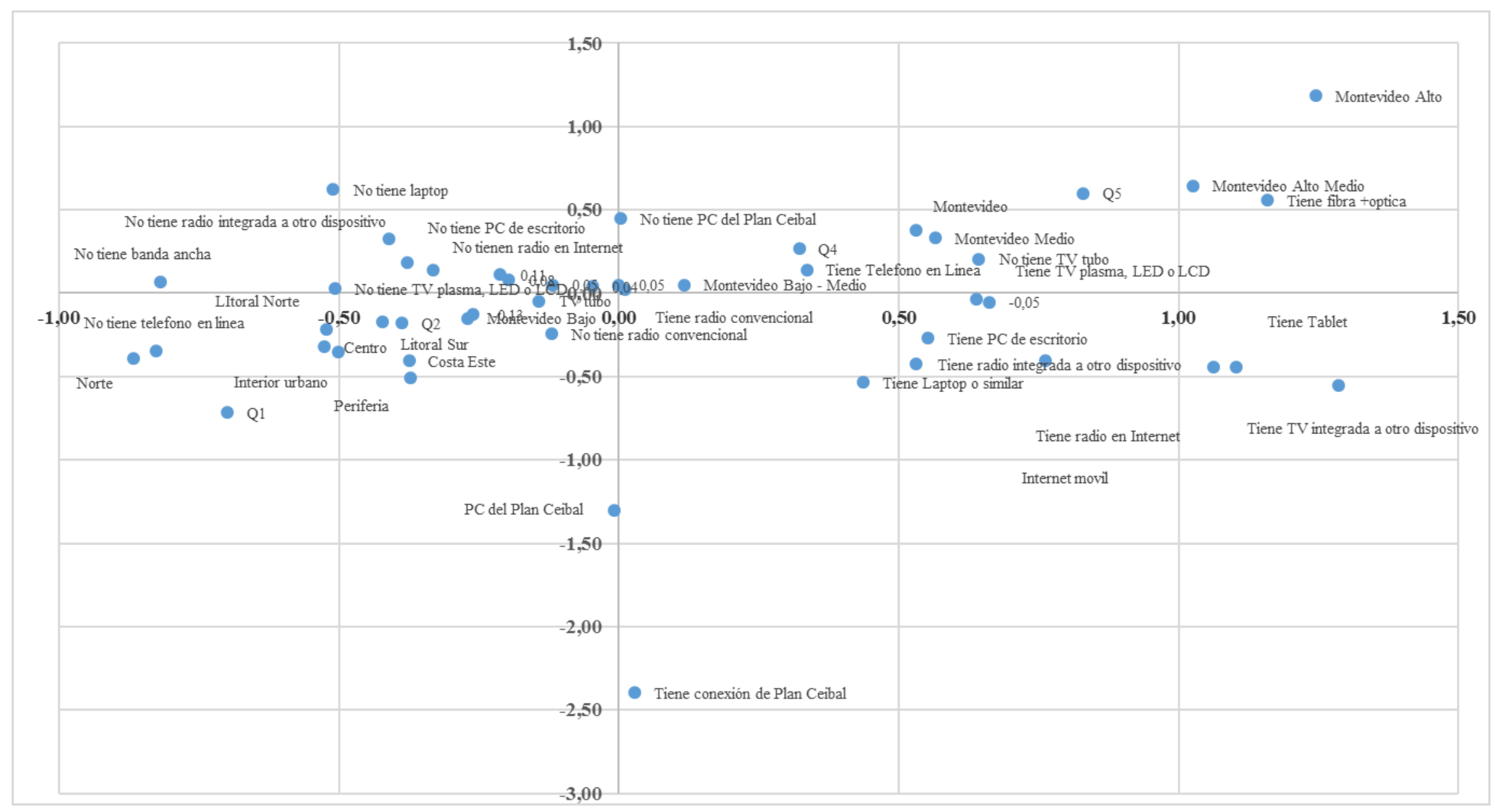

Fuente: Procesamiento propio en Base EUTIC 2013 
Perfiles de hogares a partir de conglomerados.

A los efectos de caracterizar los hogares y definir los perfiles, se realizó un análisis mediante el método de Ward. Se encontraron tres conglomerados, a partir de los cuales se definieron tres perfiles de hogar.

El primer conglomerado representa un $40,6 \%$ de los hogares del país. Son unidades que pertenecen a los primeros quintiles de ingreso per cápita, $35,1 \%$ y $24,5 \%$ al quintil $n^{\circ} 1$ y $n^{\circ} 2$ respectivamente. En su mayoría se trata de hogares del Interior del país $(61,8 \%)$, lo cual corrobora la desventaja de esta región frente a la capital en su desarrollo digital a nivel de la infraestructura de sus hogares. Estas características hicieron que a este perfil de hogares se lo denominara "hogares relegados".

Otra característica a destacar de estos hogares relegados es que el 50,4\% está integrado por al menos un adulto mayor de 60 años.

Gráfico 4: Tenencia de diferentes dispositivos en el hogar, año 2013. Grupo n 1 "Hogares relegados". En porcentaje de hogares

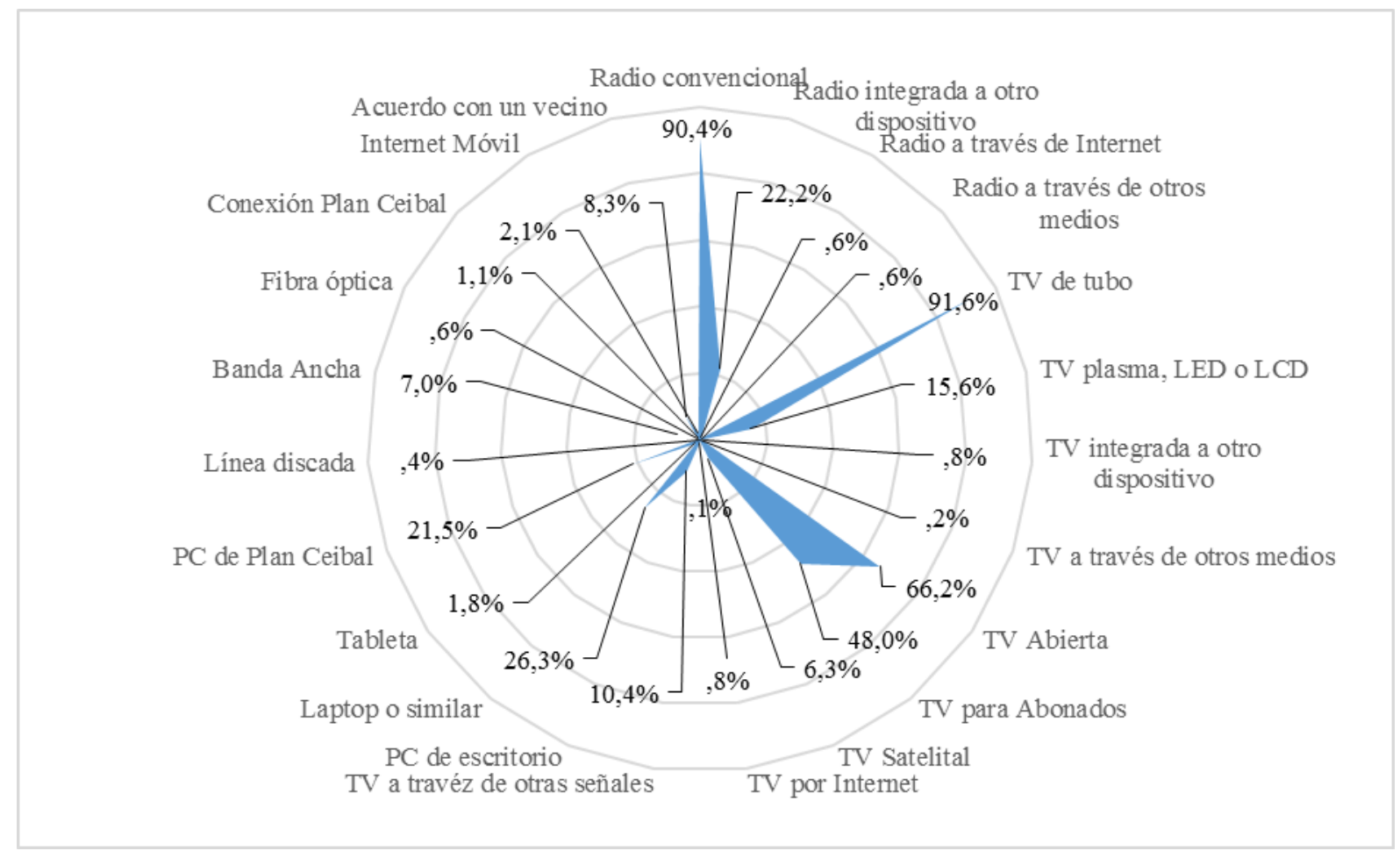

Fuente: Procesamiento propio en base EUTIC 2013.

Como se evidencia en los datos, estos hogares se encontrarían en una era de infraestructura analógica. Disponen de radio convencional el $90,4 \%$, de TV de tubo y acceden a señal abierta el $66,2 \%$ y de TV por cable un $48 \%$. Los bienes de tecnología digital no están presentes en estos hogares, por la salvedad de que el $21,5 \%$ de estos hogares cuenta con acceso a equipamiento o señal a través del Ceibal.

El grupo número 2 representa un $26,4 \%$ de los hogares y el $99,7 \%$ son hogares del Interior del país. Pertenecen a los quintiles de ingresos medios, siendo el $22,7 \%$ del primer quintil, $23,1 \%$ del tercer quintil y el $23 \%$ del cuarto quintil. En contraposición al primer grupo, estos hogares están "conectados". Disponen de los medios 
analógicos (TV de tubo, radio convencional) y también de otros dispositivos digitales (TV plasma 58,5\%, computador 63,1\%, Laptop 72,9\%). El gráfico $\mathrm{N}^{\circ} 5$ ilustra la tenencia de infraestructura de este grupo.

Grafico 5: Tenencia de diferentes dispositivos en el hogar, año 2013. Grupo n 2 "Hogares conectados". En porcentaje de hogares.

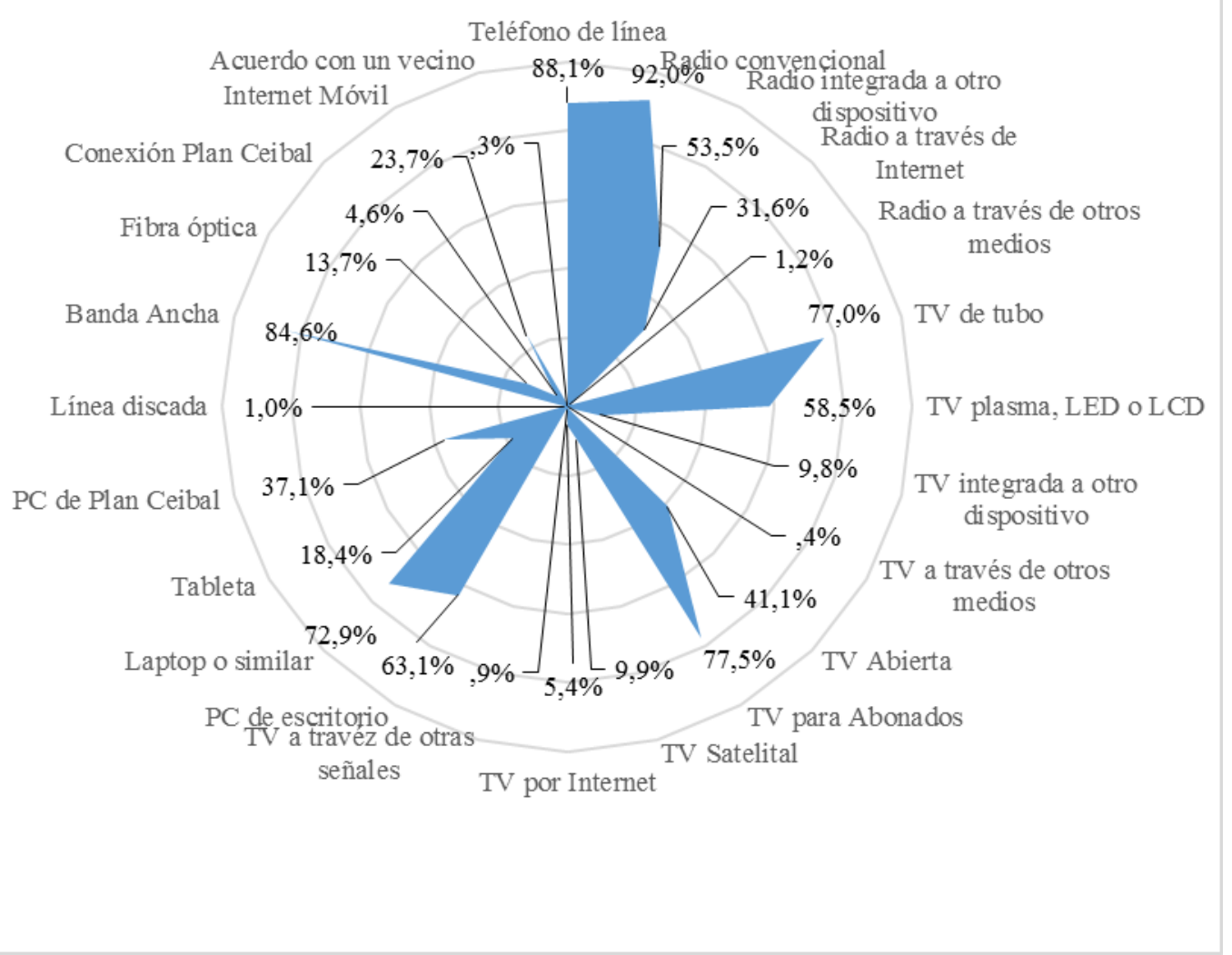

Fuente: Procesamiento propio de la EUTIC 2013.

En lo que refiere al acceso a las TIC, este grupo de hogares se encuentra relativamente "conectado". Disponen en casi un $73 \%$ de un laptop, $63,1 \%$ de computador y $84,6 \%$ de banda ancha de Internet. Son beneficiarios de la política de TIC ya que un 37,1\% accede al laptop de Ceibal, y un $23,7 \%$ a la conectividad brindada por este. Integran el uso de medios de comunicación a través de Internet, por ejemplo un 31,6\% escucha radio por la red. Por último, se encuentran aquellos hogares en la mejor situación respecto a su infraestructura. Representan un $33,1 \%$ del total de los hogares y $99,7 \%$ de los de Montevideo. Son de los quintiles de ingresos superiores (25,6\% del quintil №4; $36,8 \%$ del quintil $\left.N^{\circ} 5\right)$. Al igual que la segunda tipología de hogares, se trata de los hogares "digitales" que se encuentran en la capital del país.

Estos hogares disponen de medios analógicos en más de un 90\%, aunque también tienen dispositivos digitales como la TV plasma, o LCD $(67,8 \%)$. 
Gráfico 6: Tenencia de diferentes dispositivos en el hogar, año 2013. Grupo N³ "Digitales".

En porcentaje de hogares.

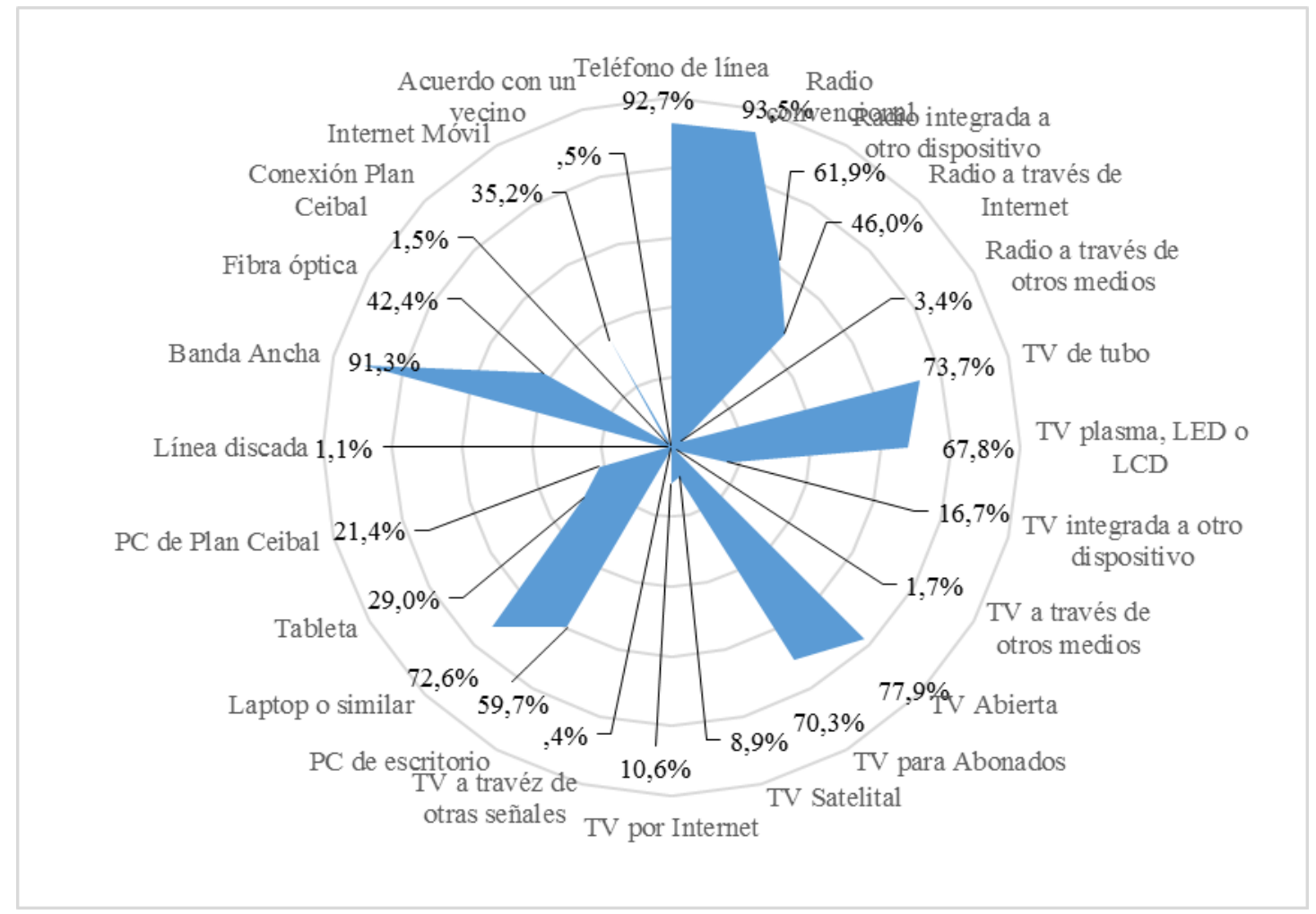

Fuente: Procesamiento propio en Base EUTIC 2013

Más del 90\% dispone de Internet por banda ancha y un 42,4\% también accede a una buena velocidad mediante conexión por fibra óptica. Los dispositivos TIC portátiles son significativos en este perfil de hogares. Un 72,6\% accede a laptop, casi $60 \%$ a PC y $29 \%$ a tableta. 35,2\% dispone de Internet móvil en este sentido.

Realizan usos de medios de comunicación tradicionales usando Internet pues el $46 \%$ escucha radio y un 10,6\% ve TV por esta vía. La política TIC no les es ajena a estos hogares, ya que un $21,4 \%$ dispone de la XO del Ceibal.

\section{Conclusiones}

En el presente artículo se analizó el equipamiento digital en hogares en Uruguay, un país que cuenta con una década de progresiva evolución de los indicadores de acceso y uso de tecnologías de la información y la comunicación (TIC) y una década de políticas públicas para la superación de la brecha digital. Con el análisis se buscó establecer la relación entre el equipamiento tecnológico del hogar, las variables estructurales, la reducción de la brecha digital y la influencia de las políticas públicas. De esta forma, se logró conocer a quienes han beneficiado las políticas de inclusión digital y qué sectores de la población permanecen fuera de su alcance.

A partir del análisis y caracterización de la expansión de la infraestructura y el acceso de los hogares, se pudo conocer las condiciones para el desarrollo informacional, las desigualdades digitales existentes y los factores asociados. Si bien el peso de la infraestructura de los hogares es un factor al que se debe relativizar el peso sobre la brecha digital, fundamentalmente debido a la aparición de dispositivos móviles, aún es importante. 
Como es sabido, el acceso a TIC en el hogar favorece su uso tanto en la frecuencia como la intensidad del mismo. De hecho la disponibilidad de señal inalámbrica en el hogar (Wifi), continúa siendo un dato de referencia importante en este contexto.

El análisis realizado, constató la importante expansión de la infraestructura de TIC en los hogares uruguayos al 2013 según la Encuesta Uruguaya de Tecnologías de la Información y la Comunicación del Instituto Nacional de Estadísticas de Uruguay. Del análisis realizado mediante la técnica de Análisis de Correspondencias Múltiples, se desprenden tres tipos de hogares que dan cuenta de perfiles de incorporación diferentes al paradigma digital. Se encuentra que las TIC han ingresado prácticamente al $60 \%$ de los hogares y que existe casi un $40 \%$ que están totalmente excluidos. Son "hogares relegados" que experimentan la brecha digital en términos de acceso a causa de su nivel socio-económico bajo. Además de estos hogares "relegados" se encuentran los "integrados" (26,4\%), los "digitales" (33,1\%). El nivel socioeconómico, la ubicación territorial del hogar y la composición demográfica se relacionan a las condiciones de infraestructura y la desigualdad digital. Los más relegados están integrados por hogares de bajos ingresos, del interior del país con personas mayores.

Estas desigualdades en la infraestructura se repiten en el análisis de los usos pues en los hogares digitales es donde más usos de los medios digitales se realizan, son los hogares de mayores ingresos, urbanos y de las edades más bajas.

A partir de estos hallazgos del análisis sobre los dispositivos TIC e internet en los hogares, confirma lo que sostuviera Mansell (2002) y Hargittai (2004) respecto a que la distribución de los beneficios del desarrollo informacional son desiguales entre los hogares. Esta persistente desigualdad digital en el acceso, vulnera las oportunidades de desarrollo informacional y por tanto de desarrollo humano para las personas de los hogares excluidos. Se confirma también, la interacción de las desigualdades sociales con las desigualdades digitales pues sus distintas variables interactúan, en lo que podríamos denominar un círculo vicioso de desigualdad. La exclusión socio-económica de estos hogares no es más que el correlato de la exclusión en infraestructura digital. Asimismo, la exclusión digital coarta a su vez la posibilidad de acceder a bienes digitales y a lograr una mejora en su nivel socio-económico.

En segundo lugar, el análisis confirma la incidencia favorable de las políticas públicas en la digitalización en todos los hogares. Esta incidencia es mayor en los más relegados pues en ellos los dispositivos, infraestructura y conectividad facilitadas por la política pública son su único medio de digitalización. Por lo que el caso uruguayo analizado estaría confirmando la centralidad de las políticas públicas específicas de acceso y conectividad. Se verifica su influencia en la inclusión digital de las personas pues sin ellas no accederían, y por tanto realizan una contribución a la reducción de la desigualdad.

A pesar de estos resultados, la incidencia de las políticas en el desarrollo tecnológico a nivel de la infraestructura de los hogares debe relativizarse. El análisis factorial realizado, evidencia que el peso del factor económico explica en mayor medida el acceso y la infraestructura digital de los hogares que las políticas TIC. El factor económico sigue determinando la tenencia de dispositivos TIC. Esto estaría dando cuenta de cierta vigencia de la distribución de dispositivos y de servicios de conectividad para las sociedades en los que estos bienes y servicios aún tienen un alto costo en relación a los ingresos de los habitantes. Confirma a la vez, las persistentes limitaciones del mercado para brindar acceso a los más amplios sectores de la población.

No obstante, el análisis brinda también elementos para relativizar la influencia positiva de las políticas TIC orientadas al equipamiento e inclusión digital de los hogares. Si bien demuestra la incidencia en el acceso del proyecto uno a uno (Ceibal) implementado a través del sistema educativo público en los sectores de menores 
ingresos, se evidencia un techo para brindar acceso a los hogares más excluidos económicamente. Si bien se reduce la desigualdad en el acceso a dispositivos lo logra la misma reducción en conectividad. Es así que la implementación de cualquier política inclusión digital debiera incluir el acceso a Internet que complete y permita el beneficio que estas tecnologías tienen para el desarrollo.

Este análisis de los hallazgos, obliga a repensar la relación entre inclusión digital y desarrollo humano dado el peso importante encontrado de las variables estructurales en la desigualdad digital. A pesar del esfuerzo realizado por las políticas públicas, los sectores más excluidos digitalmente siguen coincidiendo con los excluidos socioeconómicamente. La interacción entre la desigualdad económica y la digital, destaca la importancia de que las políticas de acceso sean políticas sociales TIC. A su vez, es importante destacar que no se trata de un único factor pues la edad de los integrantes del hogar también es un factor de exclusión que no necesariamente coincide con hogares socio-económicamente más bajos. Las personas mayores son así un sector excluido y por tanto requiere de otras medidas específicas que no se limitan al acceso a las tecnologías digitales sino también a las necesidades de los diversos sectores de la población, al acceso a internet y a medidas que favorezcan su uso con fines de desarrollo humano.

\section{Bibliografía}

Castells, M. (2000) La era de la información. Vol. 1 La Sociedad Red. Alianza Editorial. Barcelona.

Castells, M. \& P. Himanen (2014) Reconceptualizing development in the global information age. Oxford University Press.

CEPAL (2012) Cambio Estructural para la Igualdad. Comisión Económica Para América Latina, Santiago de Chile.

CEPAL (2013) Economía digital para el cambio estructural y la igualdad. Comisión Económica Para América Latina, Santiago de Chile.

Escuder, S. (2015) Desigualdades Sociales en la Sociedad de la Información: el uso de las tecnologías de la información y la comunicación en los uruguayos. Tesis de Maestría. Departamento de Sociología, Universidad de la República.

Gascó-Hernández, M., F. Equiza-Lopez \& M. Acevedo-Ruiz (2007) Information Communication Technologies and Human Development: Opportunities and Challenges. Idea Group Publishing.

Guerra, M. \& V. Jordán (2010) Políticas públicas de la Sociedad de la Información en América Latina: cuna misma visión? Documento de Proyecto, marzo de 2010, Comisión Económica Para América Latina, Santiago de Chile.

Hargittai, E. (2004) Internet access and use in context, New Media and Society, 6(1): 115-21.

Katz, R. (2009) El papel de las TIC en el desarrollo. Propuesta de América Latina a los retos económicos actuales. Ed. Ariel. Fundación Telefónica, http://www.fundacion.telefonica.com/debateyconocimiento/media/publicaciones/papel_tic_desarrollo.pd $f$

Katz, R. (2013) Banda ancha, digitalización y desarrollo. En: Jordán, V., H. Galperin y W. Peres (Coord.) Banda ancha en América Latina: más allá de la conectividad. Comisión Económica para America Latina/ Dialogo Regional sobre Sociedad de la Información (DIRSI). Santiago de Chile. 
Katz, R. \& H. Galperin (2013) La brecha de demanda: determinantes y políticas públicas. En: Jordán, V., H. Galperin y W. Peres (Coord.) Banda ancha en América Latina: más allá de la conectividad. CEPAL, DIRSI. Santiago de Chile.

Katz, R. (2015): El ecosistema y la economía digital en América Latina. CEPAL. Editorial. Fundación Telefónica. Disponible en: https://repositorio.cepal.org/bitstream/handle/11362/38916/ecosistema_digital_AL.pdf;jsessionid=E311 9FA2F1068F1B54BA2F4387BA6C0D?sequence=1 (Consultado: 18/09/2018).

López-Roldán, P.; Fachelli, S. (2013) Análisis de correspondencias. Manual de Diapositivas. Bellaterra, Universitat Autonoma de Barcelona.

Mansell, R. (2002) From Digital Divides to Digital Entitlements in Knowledge Societies. Current Sociology, Vol. 50, No. 3, 407-426.

Peña - López, I. (2009) Midiendo el Desarrollo Digital para las Políticas Públicas: El papel del Gobierno. Ponencia presentada en la II Conferencia Internacional sobre Brecha Digital e Inclusión Social, Madrid, del 28-30 de octubre de 2009. En: http://ictlogy.net/articles/20091029_ismael_pena-lopez__midiendo_desarrollo_digital_para_politicas_publicas.pdf (Consultado 18/9/2018)

Peres, W. \& M. Hilbert (Editores) (2009) La sociedad de la información en América Latina y el Caribe. Desarrollo de las tecnologías y tecnologías para el desarrollo. International Development Research Centre (IDRC); Europe AID; CEPAL. Santiago de Chile, 2009. https://repositorio.cepal.org/bitstream/handle/11362/2537/1/S0900902_es.pdf (Consultado 18/9/2018)

Rivoir, A. (2013) Enfoques dominantes en las estrategias para la Sociedad de la Información y el Conocimiento. El caso uruguayo 2000 - 2010. Revista de Ciencias Sociales N. 33, Montevideo. En: http://cienciassociales.edu.uy/wp-content/uploads/sites/3/2016/03/Presentaci\%C3\%B3n-Revista-CCSS33.pdf(Consultado 18/9/2018)

Rivoir, A. (2016) El modo de desarrollo uruguayo: inflexión histórica o estancamiento estructural. Informe de Investigación. En: Calderón, F. América Latina en la era de la información. Dilemas del desarrollo, el multiculturalismo y la innovación. Universidad de San Martín, Buenos Aires, Argentina.

Rivoir, A. \& S. Lamschtein (2014) Brecha Digital e inclusión social, contribuciones y dilemas de las políticas 1 a 1. El caso de Ceibal en el Uruguay. Razón y Palabra, v.: 87, 2. México. http://www.razonypalabra.org.mx/N/N87/V87/25_RivoirLamschtein_V87.pdf (Consultado 18/9/2018)

Rivoir, A. \& S. Escuder (2015) Múltiples escenarios de la brecha digital: Explorando perfiles de Internautas en Uruguay. En: El Uruguay desde la Sociología No 13. Pp. 125 - 146. Disponible en: http://cienciassociales.edu.uy/departamentodesociologia/wp-content/uploads/sites/3/2015/12/LibroUruguay-desde-la-Sociolog\%C3\%ADa-131.pdf (Consultado 18/9/2018).

Unión Internacional de Telecomunicaciones (2014) Informe sobre medición de la Sociedad de la Información. Ginebra. Suiza. Disponible en: https://www.itu.int/en/ITUD/Statistics/Documents/publications/mis2014/MIS_2014_Exec-sum-S.pdf (Consultado 18/9/2018) 